\title{
An Experimental Study of Natural Convection in Porous Media heated from below
}

P. Nagaraju*

Abstract

The present experimental study is carried out using a rectangular cavity packed with porous medium such as iron balls or glass balls of different diameters and hence different porosity. The lower plate is maintained at a uniform temperature $T_{h}$, which is higher than the temperature $T_{c}$ of upper plate. The experiment is performed for both closed and open systems in vertical as well as horizontal orientation of the test box. When $\left(T_{h}\right.$ $\mathrm{T}_{\mathrm{c}}$ ) is increased beyond a certain value, convective motion takes place in the fluid. The results of the experiment indicate that a porous medium can transport more energy than a saturated fluid alone if the porous matrix is highly permeable and thermal conductivity of solid particles is greater than that of fluid. This experiment also shows that convective effect is more in open system i.e., free boundary at the top. It is also observed that the time taken to reach the thermal steady state is less in the horizontal orientation as compared to the vertical orientation. In this study, the relationship between Rayleigh and Nusselt numbers has been investigated in addition to the expected temperature profile. It is found that the magnitude of temperature for iron balls is about $10 \%$ more as compared to glass balls and about $18 \%$ higher for vertical orientation as compared to horizontal orientation.

* Department of Physics, Vijaya College, Bangalore-560 004; godhundi@yahoo.co.in 
Keywords: Natural convection, Porous Medium, Heat Transfer, Rayliegh Number, Nusselt Number

\section{Introduction}

Natural convection occurs as a result of density inversion caused by either the thermal expansion of a fluid or the concentration gradients within the fluid system. The phenomenon of thermal convection through a porous layer has been observed so much in the natural world. For example; dispersion of heat through the porous layer composed of volcanic debris by natural convective movement of hot water under the ground, heat transmission from the ground (high temperature) to the atmosphere (low temperature) through a snow layer by natural convection of moist air, ground water pollution transport, nuclear waste repositors, solar collectors and petroleum reservoirs [1-2]. One of the simplest practical applications of the heat transfer theory is natural convection of liquids in vessels. Natural convection on very large scale occurs both in the atmosphere and within the earth. The phenomenon has long been of interest to geophysicists, astrophysicists and meteorologists. In petroleum reservoirs, natural convection occur as a consequence of geothermal gradients, thermal stimulation of a reservoir by steam or segregated forward combustion [3]. In all such situations it is highly beneficial to be able to predict the onset of natural convection within the reservoir and the contribution of the convective currents to the transfer of heat from one region to the other. Natural convection heat transfer has been a reliable, cost effective cooling method for the fastgrowing electronic industry where hundreds of thermal connection modules are accommodated on a small base. As the density of these heat producing module increases day by day for more compactness, the heat released should be transferred from the surface not only to protect the unit from heat but also for longer life. Natural convection can also take place in porous medium saturated with a fluid when the medium is heated from below. In recent years, scientists have focused their attention on thermal insulation and heat storage using porous media. Low density insulating materials [4] are being used increasingly in consumer appliances, clothing, homes, automobiles, aircraft and industrial 
process equipment because of savings both space and weight. Usually more than $95 \%$ of their volume is occupied by gas. Typical solid materials now being employed in these insulations are polysterene, polyurethane, wood fiber and glass. Other investigators [5-6] extended the problem to compact heat exchanger experiments, gas turbines regenerator design and absorption in granular solids. In developing a porous heat storage of supply such as solar or geothermal energy it is necessary to obtain information about the transient characteristics of heat transfer in the porous layer. The purpose of the present study is to examine experimentally the transient and steady state characteristics of convective heat transfer in the presence as well as in the absence of porous medium. Though this problem has been done by several authors [7-8], no simple and low cost experiment has appeared in the literature. The present work highlights the comparison between vertical and horizontal orientations of the test section. The analysis is based on a one dimensional treatment with uniform bulk fluid velocities and temperatures at any cross-section normal to the direction of the flow. Heat transfer due to radiation and axial conduction are neglected and internal heat generation and chemical and/or nuclear reactions are assumed to be nonexistent.

\section{Theory}

It is known that the free or natural convection takes place in the still fluid due to its heating by the contact with the hot body. If a layer of fluid is heated from below, then the density of the bottom layer becomes lighter than the top. This results in variation of temperature, density and pressure between the bottom and the top plane. Therefore uniform linear gradient of density and temperature will be established. For sufficiently small values of the temperature difference $\left(T_{h}-T_{c}\right)$ between the lower and upper plates, the viscous force overcomes the buoyancy force and the fluid remains motionless. Suppose the temperature difference $\left(T_{h}-\right.$ $\mathrm{T}_{\mathrm{c}}$ ) is increased beyond a certain value the buoyancy force overcomes the viscous force. Then the top heavy state becomes unstable and convective motion starts. The first intensive experiment was carried out by Benard in 1900. He observed the 
appearance of hexagonal cells when the instability occurs in the form of convection and it was Rayleigh who developed the theory for the instability in 1916. As a matter of fact, the gravity pulls the cooler denser fluid from the top to the bottom but the buoyancy force pushes the lighter fluid upwards. According to Rayleigh, the gravitational force between the fluid layer is opposed by the viscous damping force of the fluid. The balance of these two forces is expressed by a non-dimensional parameter called the Rayleigh number, the Rayleigh number for the case of fluid medium is

$$
\mathrm{R}_{\mathrm{af}}=\frac{\mathrm{g} \beta\left(\mathrm{T}_{\mathrm{h}}-\mathrm{T}_{\mathrm{c}}\right) \mathrm{H}^{3}}{\mathrm{va}}
$$

Here, $\mathrm{T}_{\mathrm{h}}$-Temperature of hot wall, $\mathrm{T}_{\mathrm{c}}$-Temperature of the cold wall, H-height of the cavity, $\beta=1 / T_{c}$-Coefficient of thermal expansion, $\mathrm{v}$-Kinematic viscosity, $\mathrm{g}$-acceleration due to gravity, $\mathrm{a}$ $\left(=\lambda_{e} / \rho C_{p}\right)-$ Thermal diffusivity, $\rho$ - density of fluid, $C_{p}-$ specific heat capacity of fluid at constant pressure, $\lambda_{e}$ - thermal conductivity of the mixture of fluid and solid or effective thermal conductivity.

$$
\lambda_{\mathrm{e}}=\varepsilon \lambda_{\mathrm{f}}+(1-\varepsilon) \lambda_{\mathrm{s}}
$$

where, $\varepsilon$ - porosity, $\lambda_{\mathrm{f}}$ - thermal conductivity of fluid and $\lambda_{\mathrm{s}}-$ thermal conductivity of solid. Nevertheles, the modified Rayleigh number $\left(R_{a}\right)$ in the case of a porous medium is

$$
\mathrm{R}_{\mathrm{as}}=\frac{\mathrm{g} \beta\left(\mathrm{T}_{\mathrm{h}}-\mathrm{T}_{\mathrm{c}}\right) \mathrm{HK}}{\mathrm{va}}
$$

where, $\mathrm{K}$ is the permeability of the porous medium and it is given by

$$
K=\frac{\varepsilon^{3} d_{p}^{2}}{180(1-\varepsilon)^{2}}
$$

One should note that as $R_{a}$ increases, the gravitational force becomes more dominant. At a critical Rayleigh number about 1700, the instability sets in and convection cells appear. The critical Rayleigh number can be obtained analytically for a number of different boundary conditions by doing a perturbation analysis on the linearized equations in stable state. In the case of a rigid boundary at the bottom and a free boundary at the top (without a 
lid) the critical Rayleigh number comes out as 1100. The other important parameters, which deal with natural convection are $\mathrm{G}_{\mathrm{r}}-$ Grashof number, $\mathrm{R}_{\mathrm{e}}$-Reynolds number, $\mathrm{P}_{\mathrm{r}}$-Prandtl number and $\mathrm{Nu}$-Nusselt number.

$$
\begin{gathered}
G_{r}=\frac{\rho U^{2} \times \rho g \beta\left(T_{h}-T_{c}\right) H^{3}}{(\mu U)^{2}}=\frac{g \beta\left(T_{h}-T_{c}\right) H^{3}}{v^{2}} \\
R_{e}=\frac{U H}{v}, P_{r}=\frac{v}{a}, N u=\frac{h \Delta T}{K \Delta T / H}=\frac{h H}{K}
\end{gathered}
$$

If $\frac{G_{r}}{R_{e}^{2}}>>1$, then natural convection occurs.

In natural convection, flow velocities are produced by the buoyancy forces only, hence there are no externally induced flow velocities. As a result, the Nusselt number does not depend on the Reynolds number. $R_{a}=G_{r} P_{r}=G_{r}$ (since $P_{r} \approx 1$ for gases). $N u=f\left(G_{r}\right)$ $=\mathrm{f}\left(\mathrm{R}_{\mathrm{a}}\right)$. Infact, most free convective flows arise from instabilities created by heated fluid rising past and cooler fluid must descend. It can be observed that $\mathrm{Nu}$ remains equal to 1 below the critical Rayleigh number, but increases above unity with the onset of convection. For values of $R_{a}$ not too much in excess of the critical value, convection always occurs in a fairly regular pattern. The individual elements in such a pattern are known as cells and the flow is called cellular. The geometry of the pattern can take a variety of forms such as rolls, triangular, rectangular, hexagonal and square cells with increase in $R_{a}$.

\section{Experimental apparatus and Procedure}

The experiment is performed by using a rectangular wooden box of width $95 \mathrm{~mm}$, depth $99 \mathrm{~mm}$ and height $628 \mathrm{~mm}$. But the cross sectional area of the test section is $85 \mathrm{~mm} \times 89 \mathrm{~mm}$. 
Picture given here shows the Experimental Apparatus. The main parts of the apparatus consist of heating and cooling parts along with porous media packed inside the box. Surface temperature of the hot wall (copper plate $10 \mathrm{~mm}$ in thickness) is maintained by using three strip heaters (soldering iron), each having a maximum capacity of $25 \mathrm{~W}$.

Actually two soldering rods are inserted on one side of the plate

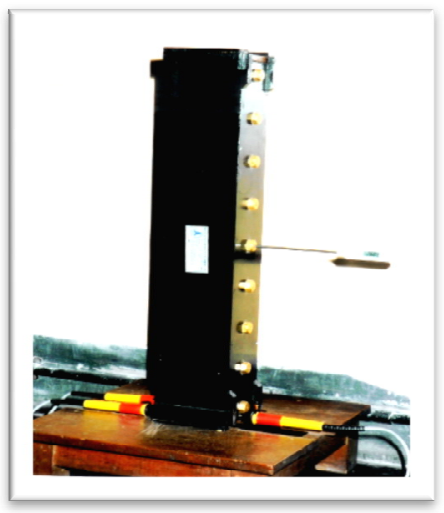

Figure 1 almost at its ends and on the opposite side one more soldering (third one) is inserted into the middle of the plate. Thus it is ensured that the bottom plate is uniformly heated and maintained at $T_{h}$, and the surface temperature of the cold wall $T_{c}$ (copper plate $10 \mathrm{~mm}$ in thickness) is kept at room temperature. 9 holes are made on one of the vertical walls of the box and each hole is fitted with a conical shaped cap to introduce the digital thermometer. The holes/thermometers are located at 1, 8.3, 15.3, 22.7, 29.6, 36.9, 44.2, 51.4 and $58.5 \mathrm{~cm}$ from the bottom hot plate. It is taken care that no air enters into the box or leave from the box. That is the rectangular box is made air tight with bottom and the top plates isothermal (but at different temperatures) and the side walls adiabatic.

Measurements are made for three solid particles, glass balls of diameters $15 \mathrm{~mm}$ and $10.85 \mathrm{~mm}$ and steel balls $9.5 \mathrm{~mm}$ respectively with air as the fluid. In this work, the porosity is determined by measuring the volume ratio of filled water in the packed container to the empty container. It is obtained 0.415 and 0.419 for glass balls and 0.444 for iron balls. However, for spherical solid particles, the value of $\varepsilon$ is almost unity near the wall surface due to one particle having only one contact point with the wall surface. For a packed bed as reported by Yagi et al [9], the porosity distribution from the wall surface to a distance of radius of solid particle in the direction of core portion of porous layer is varied from 1 to 0.4 and that inside of porous layer becomes constant. 
In this study, the values of some similarity parameters are:

$\mathrm{R}_{\mathrm{af}}$ (fluid) $=3.47 \times 10^{7}, \mathrm{R}_{\mathrm{as}}$ (glass balls) $=1.21 \times 10^{5}, \mathrm{R}_{\mathrm{as}}$ (iron balls) $=$ $2.14 \times 10^{3}, \mathrm{~K}$ (glass balls) $=2.34 \times 10^{-5}, \mathrm{~K}$ (iron balls) $=1.42 \times 10^{-}$ 7.This shows that the values of $R_{a}$ are very much smaller than the corresponding values for fluid alone. The experiment is conducted in a room, where the thermal disturbance from the environment is prevented. This is because the readings are likely to be affected by any cross flow of breeze (all the doors and windows of the room are closed and no fans are allowed to run). Temperature is measured at regular intervals of time till the steady state is reached. In fact, to reach the steady state, it takes about 4 hours. The experiment is also done by keeping the test section horizontal. In the case of a cavity a geometrical parameter, aspect ratio needs to be considered. When the test section is vertically oriented, aspect ratio $\mathrm{H} / \mathrm{W}=6.38$ and when it is horizontally oriented, aspect ratio $\mathrm{W} / \mathrm{H}=0.156$. The measured values for horizontal orientation is entirely different from vertical orientation. Further, the time taken to reach the steady state is less than that of the vertical orientation.

\section{Experimental results and Discussion}

To gain a perspective of the physics of heat transfer by natural convection, the experimental arrangement is made in such a way that the lower boundary at $y=0$ is maintained at a temperature $T_{h}$ and the upper boundary $y=H$ is at $T_{c}$, with $T_{h}>T_{c}$. But, $T_{c}$ is dependent on the medium in the enclosure. The porous medium has scalar permeability $K$ and is saturated with fluid of density $\rho$ and viscosity $\mu$. We now consider the test section as described above and shown in Fig.1. The height of the vertical channel is $\mathrm{H}$, while separation is $\mathrm{W}$. It is noted that the temperature near the heated wall increases because of the heating effect from the wall(bottom). At the other end, the temperature near the cold wall(top) decreases because of the cooling effect from the surroundings. Figure 2 shows that the typical non-dimensional temperature distribution $(\theta)$ for the aspect ratio of $\mathrm{H} / \mathrm{W}=6.38$ and the width of cavity $\mathrm{W}=95 \mathrm{~mm}$. In what follows is the temperature distribution in the case of vertical section filled with iron balls or glass balls as porous medium and absence of porous medium (air 
alone). The temperature distribution for iron balls and glass balls are similar, but the magnitude of temperature for iron balls is more, about $10 \%$ at $0.137(y=8.3 \mathrm{~cm})$ as compared to glass balls. However, in the absence of porous medium, the magnitude of temperature is more by about $7 \%$ as compared to glass after $8.3 \mathrm{~cm}$. The reason for more temperature distribution in the presence of iron balls near the hot wall indicates that the intensity of the convection is weak due to the large thermal conductivity.

Figure 3 shows the temperature distribution in both the vertical and horizontal orientation of the test section. The magnitude of the temperature in the presence of glass balls in the horizontal orientation is of little attention. However, in the absence of porous medium, there is considerable difference between the vertical and horizontal orientations. In fact, magnitude of the temperature in the vertical orientation is about $18 \%$ higher (at 0.016 or $y=1 \mathrm{~cm}$ ) than the horizontal orientation. Nevertheless, it keeps decreasing as one approaches the cold wall.

Figure 4 exhibits the temperature profile $(\theta)$ for the aspect ratio 6.38 and glass balls of different diameters. It is found that there is no much difference in magnitude of temperature even though the diameters are different.

\begin{tabular}{|c|c|c|c|c|c|c|c|c|c|}
\hline $\mathrm{y} / \mathrm{H}$ & 0.016 & 0.137 & 0.252 & 0.374 & 0.488 & 0.609 & 0.729 & .848 & 0.965 \\
\hline Air & 2.85 & 1.08 & -0.21 & -0.12 & -0.02 & 0.23 & 0.4 & 0.83 & 1.53 \\
\hline Iron balls & 1.62 & 0.86 & 1.88 & 0.73 & 0.17 & 0.04 & -0.05 & 0.0 & 0.0 \\
\hline
\end{tabular}

Table 1: Percentage variation of $\theta$ for air and Iron balls

Table 1 shows the variation of dimensionless temperature $\theta$, when the upper boundary is changed from a rigid-lid to a constant pressure surface. Removal of the lid leads to enhanced convective motion because the hot plume free to leave the system and its width narrower. Where as, in the confined case the cold plume emerges from a thermal boundary layer along the top surface. As such, descending fluid is generally warmer than in the case of a permeable top, where all fluid entering the porous medium is at the temperature of the cold upper surface. 


\begin{tabular}{|c|c|}
\hline $\mathrm{R}_{\mathrm{a}}$ & $\mathrm{Nu}$ \\
\hline $3.47 \times 10^{7}$ & 749.6 \\
\hline $1.22 \times 10^{5}$ & 51.0 \\
\hline $2.14 \times 10^{3}$ & 7.86 \\
\hline
\end{tabular}

Table 2: Nusselt number and Rayleigh number

The results of this experiment is compiled in terms of the usual dimensionless groups, namely, the Nusselt number and the Rayleigh number. Table 2 shows the relationship between the steady state Nusselt number and the Rayleigh number for the systems investigated.

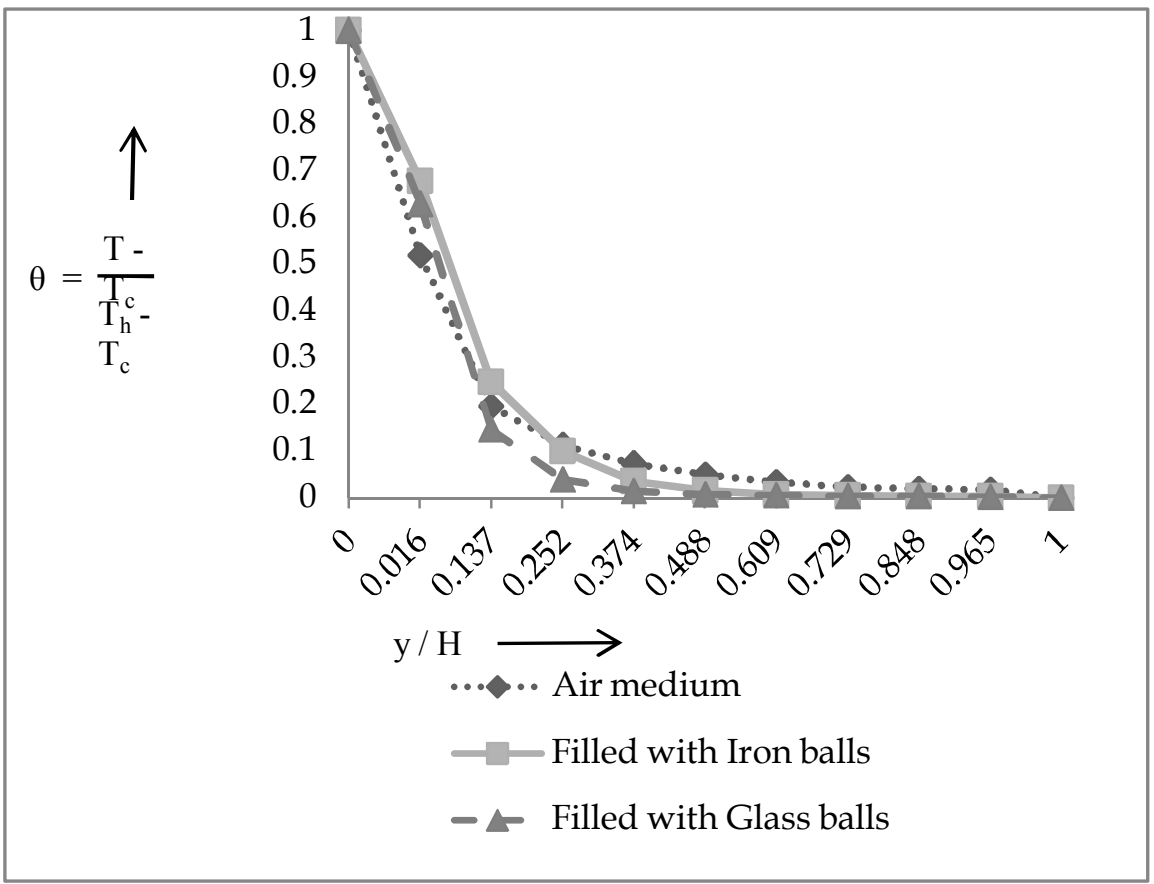

Figure 2: Temperature distribution in a vertical porous layer for $\mathrm{W}=95$ $\mathrm{mm}, \mathrm{H} / \mathrm{W}=6.38 \mathrm{~T}_{\mathrm{h}}=260^{\circ} \mathrm{C}, \mathrm{T}_{\mathrm{c}}=30^{\circ} \mathrm{C}$ (for air medium), $\mathrm{T}_{\mathrm{c}}=25^{\circ} \mathrm{C}$ (for glass and iron balls)

\section{Conclusion}

The experiment is carried out by the rectangular cavity packed with/without porous media, whose opposing horizontal walls 
have different but uniform temperatures and the other walls are adiabatic. The following conclusions are obtained.

1. An increase of the natural convection in the porous layer causes a shorter time to reach a steady state and thermal homogeneous in the porous medium

2. A porous medium can transport more energy than a saturated fluid alone if the permeability and thermal conductivity of solid particles is greater than that of fluid

3. The magnitude of the temperature in the vertical orientation is about $18 \%$ higher than that of the horizontal orientation.

\section{References}

[1] D.A. Nield and A. Bejan. Convection in Porous Media, 2nd ed, Springer, New York, 1998

[2]D.B. Ingham and I. Pop (Eds)., Transport Phenomena in Porous Media, Pergamon Press, Oxford, 1998.

[3]Vafai. K., Handbook of Porous Media. Marcel Dekker, New York 2000.

[4]Larkin B. K, Churchill S.W., Heat transfer by radiation through Porous insulations, AIChE Journal 5 467-474, 1959.

[5]Pop. I, Ingham D.B., Convective heat transfer, Mathematical and computational modeling of viscous fluids and porous media, Pergamon, Oxford, 2001.

[6]M. N. Ozisik., Heat transfer, McGraw Hill, 1986

[7]Nobuhiro Seki, S. Fukusako and Inaba., Heat transfer in a confined rectangular cavity packed with porous media. Int J. Heat transfer and Mass transfer, 21-11 (1978), 985

[8]H. Inaba, N. Seki., A study on transient characteristics of Natural Convective Heat transfer in a confined rectangular cavity packed with porous media, Bulletin of JSME, Vol 26, No. 222, 1983.

[9]S. Yagi and D. Kunii., Studies on heat transfer in packed beds, Int Dev, Heat transfer, 4, 750, 1962. 
Mapana J Sci, 10, 2(2011) Natural Convection in Porous Media heated from below

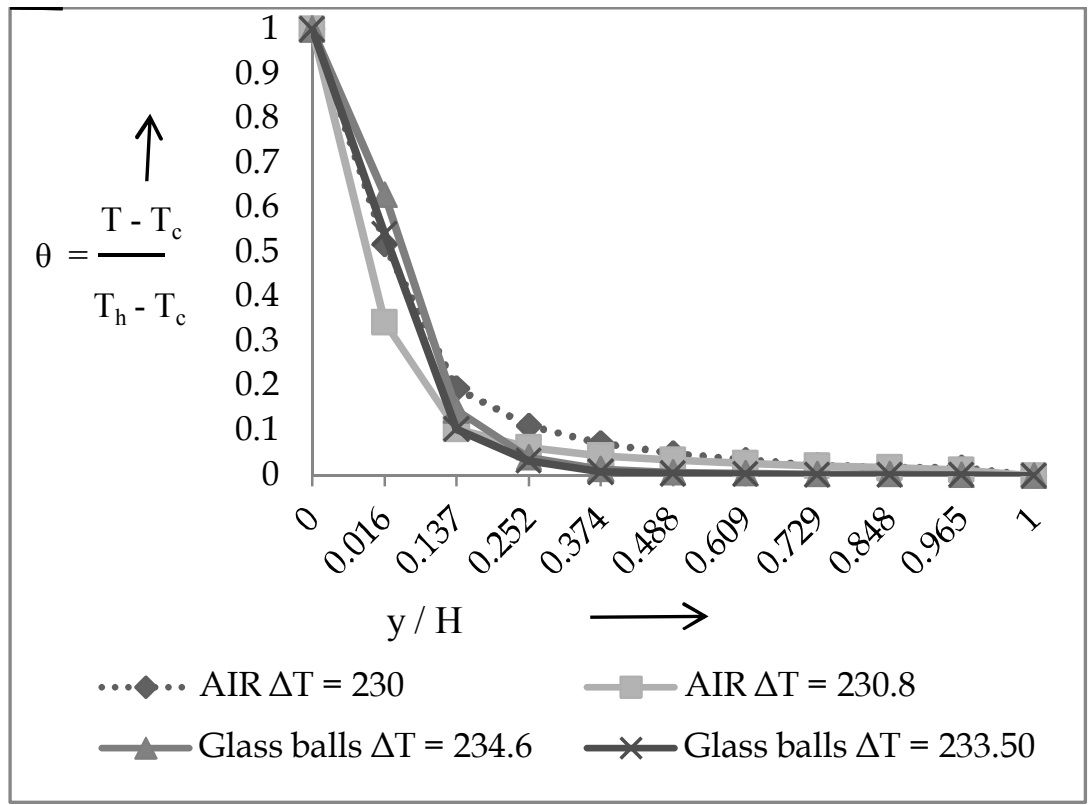

Figure 3: Temperature distribution in the vertical and Horizontal porous layer for $\mathrm{W}=95 \mathrm{~mm}, \mathrm{~W} / \mathrm{H}=0.156, \mathrm{~T}_{\mathrm{h}}=260^{\circ} \mathrm{C}, \mathrm{T}_{\mathrm{c}}$ (Vertical, air) $=30^{\circ} \mathrm{C}, \mathrm{T}_{\mathrm{c}}$ (Horizontal, air) $=29.2^{\circ} \mathrm{C}, \mathrm{T}_{\mathrm{c}}$ (Vertical, glass balls) $=25^{\circ} \mathrm{C}, \mathrm{T}_{\mathrm{c}}$ (Horizontal, glass balls) $=26.5^{\circ} \mathrm{C}$ 


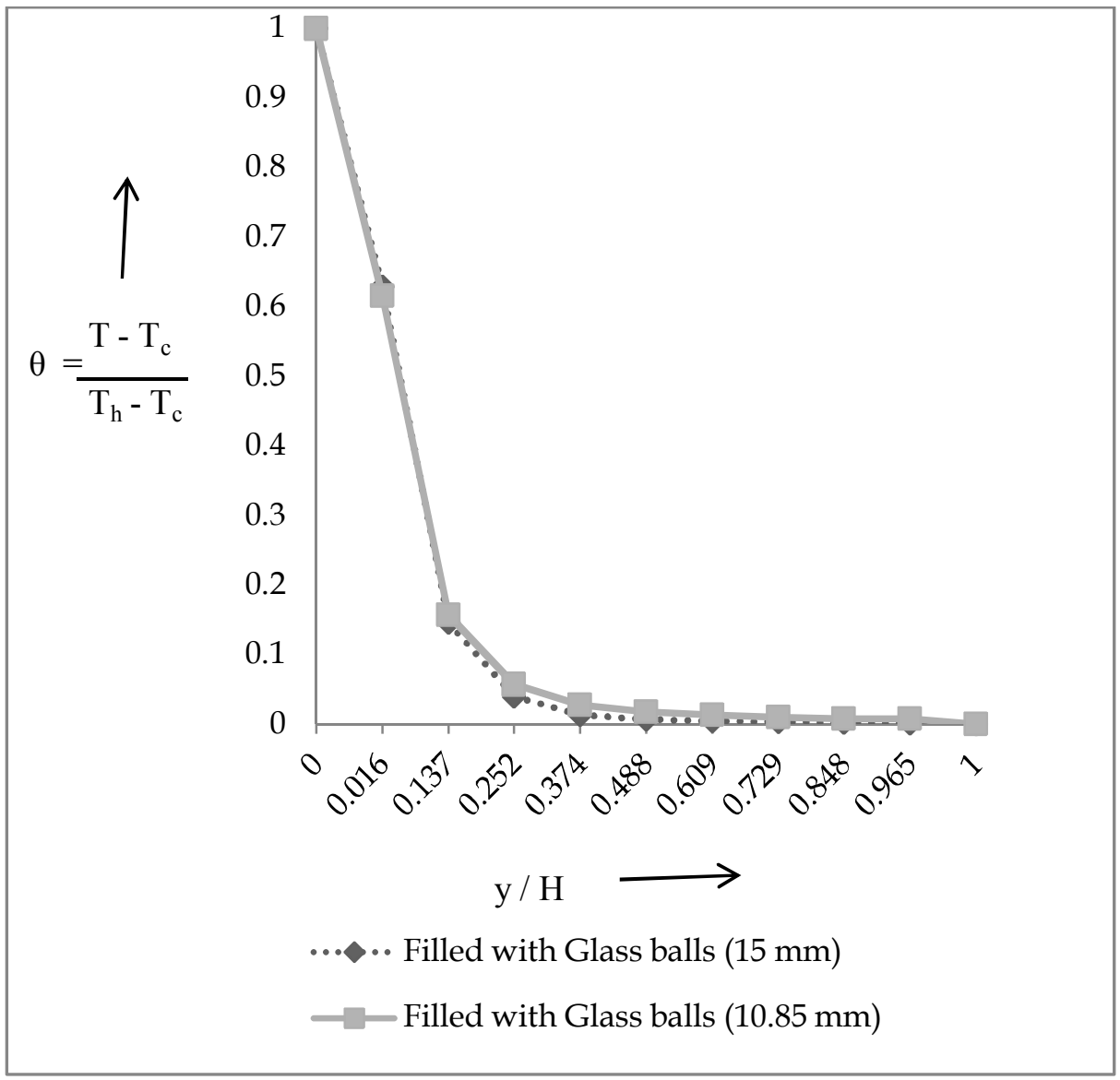

Figure 4: Temperature distribution in a vertical porous layer for $\mathrm{W}=95$ $\mathrm{mm}, \mathrm{H} / \mathrm{W}=6.38, \mathrm{~T}_{\mathrm{h}}=260^{\circ} \mathrm{C}, \mathrm{T}_{\mathrm{c}}=25^{\circ} \mathrm{C}$ 